\title{
Gas Phase Nanoparticle Integration
}

\author{
Chad R. Barry and Heiko O. Jacobs* \\ Department of Electrical and Computer Engineering, University of Minnesota, 200 Union \\ Street SE, Minneapolis, Minnesota 55455, hjacobs@umn.edu
}

\begin{abstract}
We report on two gas phase nanoparticle integration processes to assemble nanomaterials onto desired areas on a substrate. We expect these processes to work with any material that can be charged. The processes offer selfaligned integration and could be applied to any nanomaterial device requiring site specific assembly. The Coulomb force process directs the assembly of nanoparticles onto charged surface areas with sub-100 nm resolution. The charging is accomplished using flexible nanostructured electrodes. Gas phase assembly systems are used to direct and monitor the assembly of nanoparticles onto the charge patterns with a lateral resolution of $50 \mathrm{~nm}$. The second concept makes use of fringing fields. The fringing fields directed the assembly of nanoparticles into openings. The fringing fields can be confined to sub $50 \mathrm{~nm}$ sized areas and exceed $1 \mathrm{MV} / \mathrm{m}$, acting as nanolenses. Gas phase assembly systems have been used to deposit silicon, germanium, metallic, and organic nanoparticles.
\end{abstract}

Keywords: nanoparticle, gas phase, electrostatic, assembly, integration

\section{INTRODUCTION}

Nanomaterials are considered the building blocks of future nanotechnological devices. These nanomaterials are most commonly fabricated using solution chemistry or gas phase chemistry and can provide a variety of functions. A number of concepts have been developed to print nanoparticles directly from powder and solution. Sub - one micrometer resolution assembly has been accomplished using charge directed nanoxerographic printing $[1,2]$ and topographically directed assembly incorporating both capillary and electrostatic forces $[3,4]$. While the solution methods have emerged, directed self-assembly processes from the gas phase are not widely available but immensely important. Such processes would enable the integration of unique gas phase synthesized nanomaterials at desired locations on a substrate. The nanomaterial building blocks would not have to be transferred in solution as is currently the case. Solution concepts have their own set of problems - almost all of the reviewed receptor based assembly concepts $[1,2,5-13]$ require surface functionization to prevent agglomeration or to guide the assembly which often interferes with the electronic or optical properties. Direct integration from the gas phase would support the use of well established passivation concepts to create high quality nanomaterial building blocks that maintain the electronic and optoelectronic functionality. Two nanoparticle assembly tools are presented that make use of electrostatic directed assembly to integrate functional materials at desired locations on a surface. The processes enable localized integration directly from the gas phase without transferring the materials into solution. The concept, based on electrostatic forces, can be used to integrate any material that can hold charge, both organic and inorganic.

\section{MATERIALS AND METHODS}

The two processes, Coulomb force directed assembly and fringing field directed assembly, are illustrated in figure 1. The Coulomb force process (fig. 1a) directs the assembly of nanoparticles onto a flat substrate containing charged surface areas with sub-100 nm resolution. The fringing field directed assembly approach (fig. 1b) focuses the assembly of nanoparticles into openings in a nanostructured thin film with sub-50 $\mathrm{nm}$ resolution.

The Coulomb force directed assembly process makes use of flexible electrode structures that can form sub 100 $\mathrm{nm}$ sized electrical nanocontacts by mechanical placement of the electrode structure on a second surface. The electrical nanocontacts that are established at the interface are used to inject charge patterns in a dielectric thin film. We refer to this process as electric nanocontact printing of charge. The electrode structures used in electric nanocontact printing are made from lithographically patterned thin silicon $(10 \mu \mathrm{m}$ thick) wafers [14] and metal coated poly(dimethylsiloxane) (PDMS) stamps [1, 9]. For the dielectric substrate, we used a $60 \mathrm{~nm}$ thick film of PMMA spin coated onto a silicon wafer. The substrate chips were placed on the flexible electrodes by hand and contacted from the back with a metallic needle attached to a micromanipulator. The micromanipulator was used to adjust the needle's position and to provide pressure to establish contact between the electrode and PMMA thin film. An external voltage was applied for 30 seconds to generate a constant current of $1 \mathrm{~mA}-5 \mathrm{~mA}$. After removal, the current carrying charge is trapped at the contacted areas of the PMMA surface, generating a charge pattern matching the pattern on the electrode structure. These charge patterns can be used to direct the assembly of nanoparticles. 
The fringing field directed assembly approach is different from the Coulomb force or nanoxerographic printing method that uses continuous dielectric film layers. The primary aim of this approach is to direct single

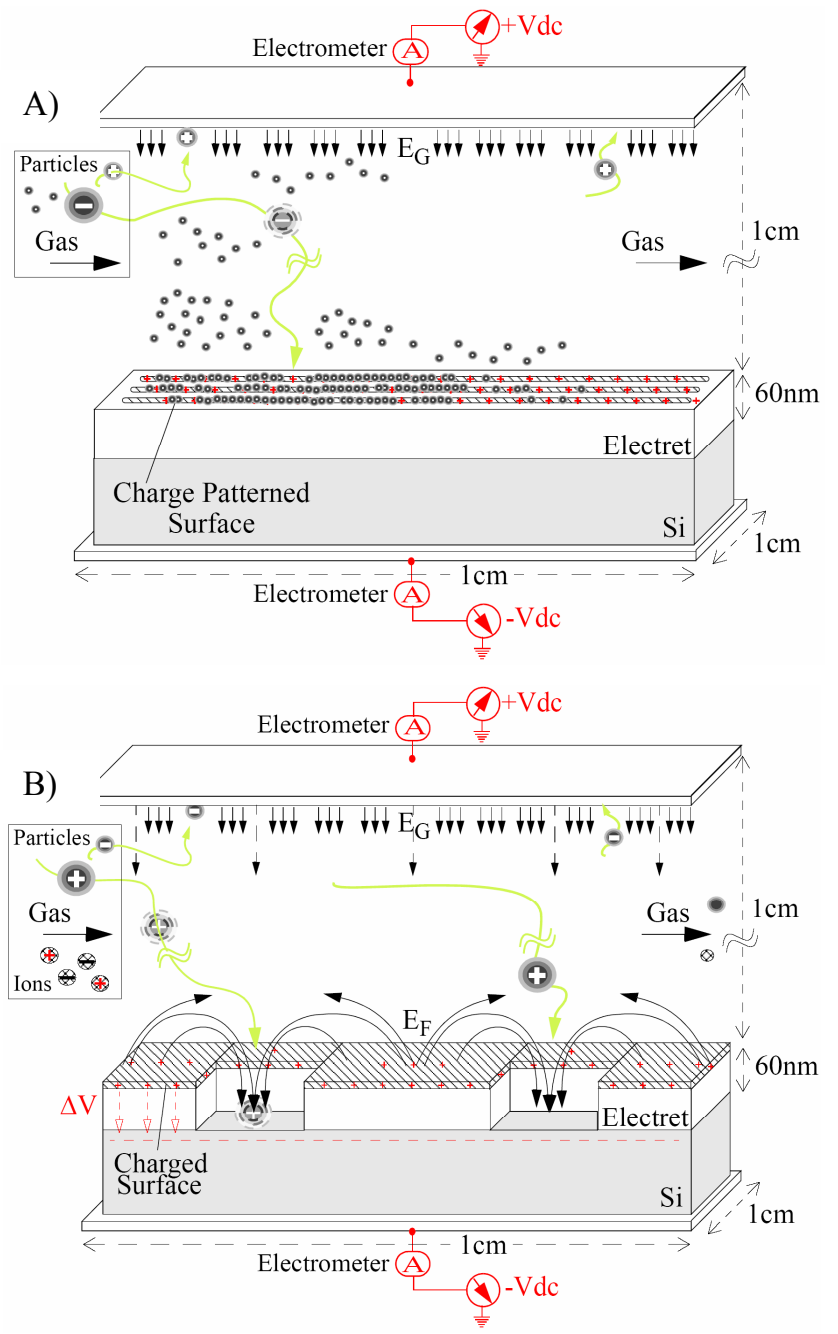

Figure 1: Illustration of the sample and assembly setups. A carrier gas delivers charged nanoparticles and ions. A global field $E_{G}$ directs charged particles of selected polarity towards the charged sample surface establishing an electrometer current. A) A Coulombic force of attraction exists between the charged particle and the charge patterned surface that directs the assembly. B) A potential $\Delta V$ is present between the electret and substrate. The potential gives rise to local fringing fields $E_{F}$ that direct nanoparticles into the openings.

nanoparticles into addressable regions on a surface. It combines Coulomb force directed assembly [10, 13, 14] with topographically patterned materials that can be formed by conventional lithography.

Figure 1 helps illustrate the fringing field directed assembly concept. A global $\left(E_{G}\right)$ field, generated within the assembly module, and localized $\left(E_{F}\right)$ electrostatic field are used to direct the assembly of charged nanomaterials into micro or nanometer sized openings. The fringing field is formed using a charged, patterned thin film on top of a silicon chip. The surface of the electret film is at a different electrostatic potential than the silicon chip. Electrostatic field lines are present not only inside the electret but also outside, affecting the nanoparticle trajectories. The externally biased assembly module electrodes select and direct incoming particles of a desired polarity towards the chip surface. Both electrodes are connected to an electrometer (keithley 6517A) to monitor the current and charge that arises when charged nanomaterials or ions deposit on the surface. In this faraday cup arrangement, image charges flow from the ground through the electrometer into the sample or electrode plate to the location of assembled material. As a result, the electrometer measures the accumulated charge of the assembled particles or ions regardless of where they deposit on the surface or whether they become neutralized by the image charges.

The substrate patterns can be generated by any number of lithography methods including nanoinprint, hot embossing, and micromolding in capillaries. In our experiments we used electron beam (e-beam) lithography to generate $100 \mathrm{~nm}-1 \mu \mathrm{m}$ wide openings (holes and lines) in a $60-80 \mathrm{~nm}$ thick layer of PMMA that was spin coated onto a silicon wafer. The PMMA on silicon exhibited an inherent built-in potential difference $(\Delta \mathrm{V})$ that can be reversed or enhanced by additional surface treatments such as contact or corona charging. This potential difference $(\Delta \mathrm{V})$ gives rise to the fringing fields and is the key parameter in the self-assembly process. The addition of a global field between the assembly module electrodes directs particles toward the sample and aids in the focusing effect. By balancing the superposition of the global and local fields, nullified areas can be generated in which there are no directing forces and therefore no assembled particles. This balanced superposition also increases the fringing field strength over the nanostructured areas. These strong electric fields can be used to focus the assembly of nanoparticles. [15]

We and others have already demonstrated three fundamentally different assembly strategies using powders of nanoparticles [9]; nanoparticles from the liquid phase [1, $11,16]$; and most recently nanoparticles from the gas phase $[10,13,14,17]$. A system that forms nanoparticles by evaporation of metals $(\mathrm{Au}, \mathrm{Ag}, \mathrm{Ga}, \mathrm{In}, \mathrm{Zn}, \mathrm{Cu})$ and semiconductor $(\mathrm{Ge})$ precursors $[10,14]$ as well as a system that forms organic protein nanoparticle aerosols from solution [17] were employed to aid in the directed assembly process. Using these systems, we were able to show 30-100 $\mathrm{nm}$ resolution site specific assembly of nanoparticles from the gas phase. $[14,15,17]$

An example of one of these systems, shown in figure 2, involves the use of a tube furnace that generates nanoparticles in the gas phase by evaporation, nucleation, and condensation. An argon carrier gas transports the 


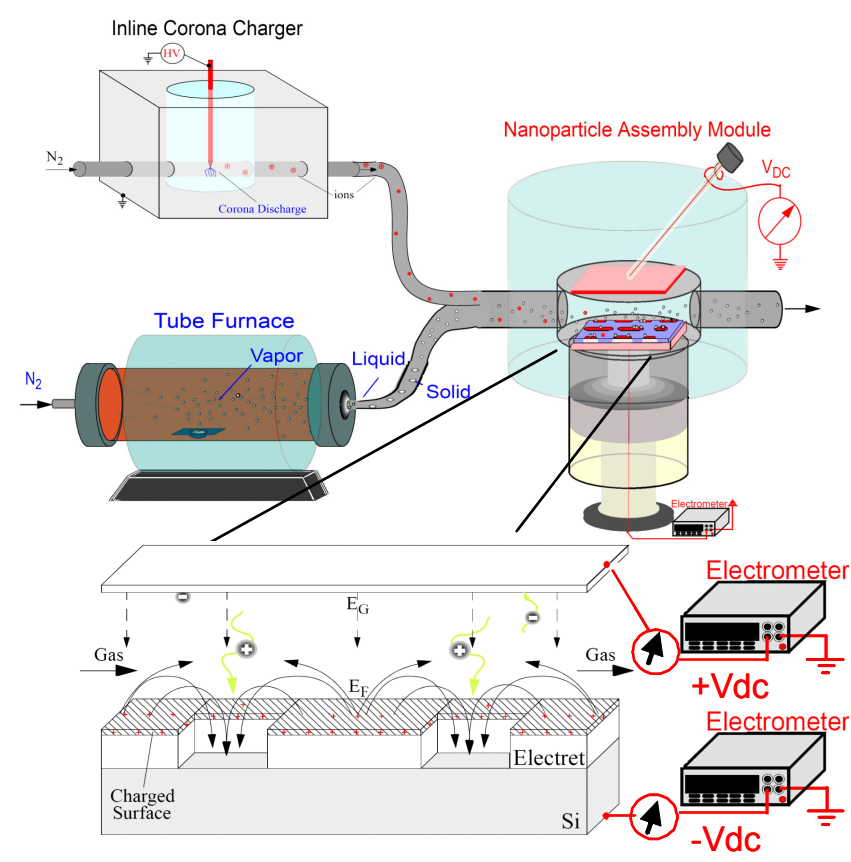

Figure 2: Gas-phase assembly system. Materials are evaporated at a high temperature and then nucleate and condense into nanoparticles as they are carried out of the tube furnace by an argon carrier gas. An inline corona charger is used to generate a high concentration of ions that are flown into the assembly module to boost the charge differential on the topographically patterned substrates.

particles out of the furnace and into the particle assembly module. The assembly module consists of a cavity that holds the sample, two electrodes to generate a global electric field, and an electrometer to count the charge of the assembled particles. The global electric field directs incoming charged particles of selected polarity towards the sample surface, where the local electrostatic force takes over and assembles the particles onto or into the patterned areas depending on the assembly process used. In addition, a corona charger was placed inline to increase the charge differential on the topographically patterned substrates. Argon gas was flown through the charger and ionized using a positive DC corona discharge by applying a positive $(\sim 2.5 \mathrm{kV})$ potential between an insulated stainless steel needle and a partially hollowed out metal encasement. A fraction of the argon ions are carried away by the gas flow into the assembly module, where they are deposited on the substrate by the global field. The deposited ions are trapped on or inside the insulating electret surface whereas the conducting silicon surface remains less affected.

\section{RESULTS}

Fig. 3 shows representative images of nanoparticles assembled from the evaporation system using both the Coulomb force directed assembly process and the fringing field process. Figure 3a shows assembly of germanium nanoparticles onto charge patterned squares consisting of $60 \mathrm{~nm}$ wide lines. The charge patterns were generated using electric nanocontact printing with the thin silicon electrodes. Figure $3 \mathrm{~b}$ shows assembly of silver nanoparticles focused into $100 \mathrm{~nm}$ holes using fringing fields created by ion deposition from the inline corona charger.

The observed focusing effect and expected precision can be studied by solving Langevin's equations of motion of nanoparticles inside an electric field. [15] The relative precision appears to be dominated by the potential difference between two material surfaces and the charge on

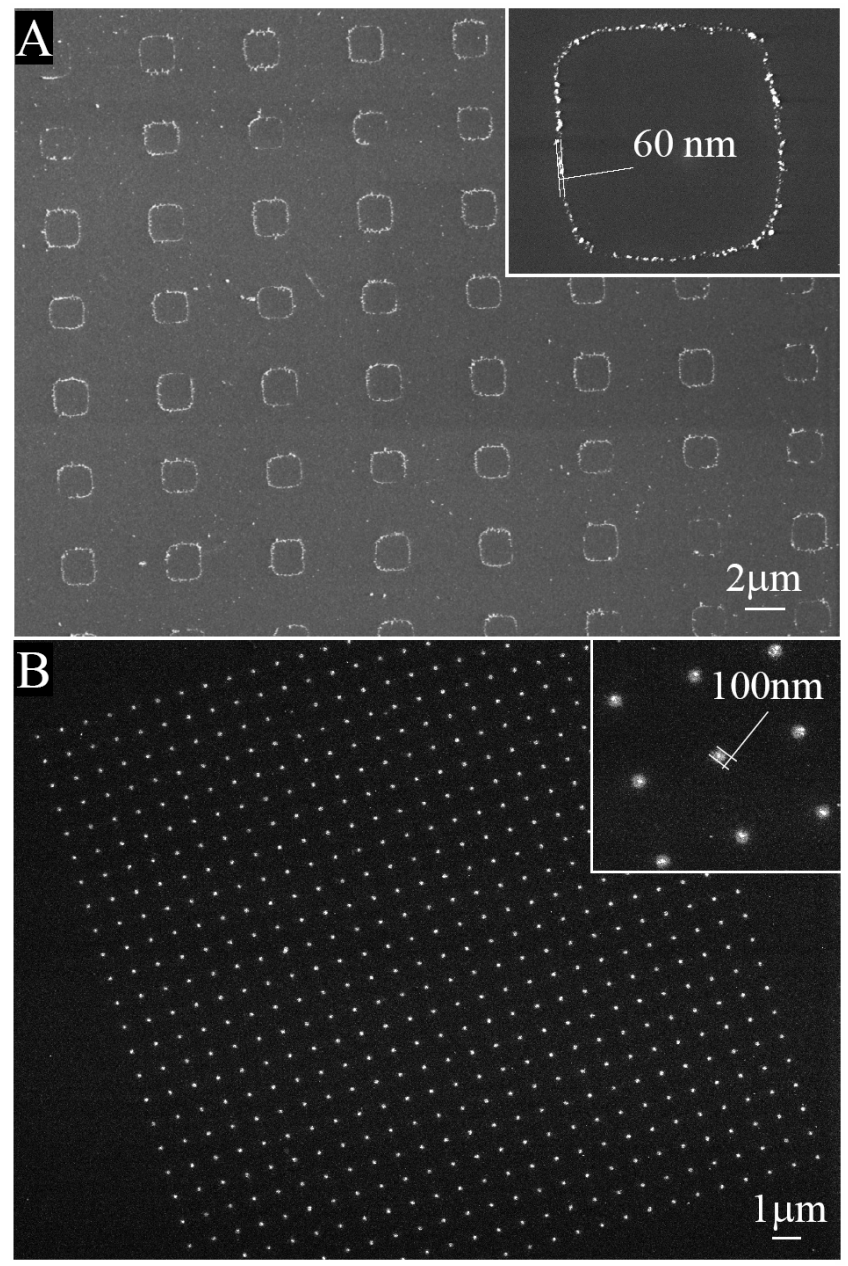

Figure 3: Scanning electron microscope images of assembled nanoparticles generated by evaporation. A) Germanium nanoparticles assembled onto charge patterned areas with $60 \mathrm{~nm}$ resolution. B) Silver nanoparticles focused into the center of $100 \mathrm{~nm}$ holes by the localized fringing fields. 
the particle in comparison to the thermal energy. Further increasing the particle charge and potential difference $(\Delta \mathrm{V})$ above the thermal energy increases the relative precision. In addition, the precision is independent of the size of the patterns and therefore scaleable.

\section{CONCLUSION}

In conclusion, thin silicon provides a suitable, semiflexible material that can form electrical nanocontacts as small as $50 \mathrm{~nm}$ by placing the nanostructured electrode onto an electret surface. The nanocontacts can be used to inject charge into $50 \mathrm{~nm}$ sized areas. The highest resolution we have obtained so far is $60 \mathrm{~nm}$, however, there appears to be no physical limitation to suggest that Coulomb force directed assembly cannot be used at the sub-10 nm length scale. While structures as small as $10 \mathrm{~nm}$ have been generated in bulk silicon [18], they are not widely available. For this reason and the novelty of the process, we have developed the fringing field directed assembly method. The focusing effect provided by this process could enable integration of materials and devices into areas $3-10$ times smaller than the focusing elements themselves. For example, the ability to integrate nanoparticles at desired locations would enable the integration of well defined nanowires (due to the size selection of the nanoparticles) when combined with existing catalytic nanowire growth concepts. These processes are directed by long range electrostatic forces that can be adjusted and controlled by controlling the charge differential between patterned and unpatterned areas or between the surfaces of two patterned materials. The processes therefore offer self-aligned integration and could be applied to site specific nanowire growth via positioned cataylst seeds, integrated single crystal silicon nanoparticle transistors, or other nanomaterial devices requiring assembly onto desired surface areas. They could also be extended to externally biased surface electrodes that could be programmed to enable the integration of more than one material type.

\section{REFERENCES}

1. Jacobs, H.O., S.A. Campbell, and M.G. Steward, Approaching NanoXerography: The use of Electrostatic Forces to Position Nanoparticles with 100 Nanometer Scale Resolution. Advanced Materials, 2002. 14(21): p. 1553-1557.

2. Barry, C.R., et al., Printing nanoparticles from the liquid and gas phases using nanoxerography. Nanotechnology, 2003. 14(10): p. 1057-1063.

3. Cui, Y., et al., Integration of Colloidal Nanocrystals into Lithographically Patterned Devices. Nano Letters, 2004. 4(6): p. 1093-1098.

4. Ma, L.-C., et al., Electrostatic Funneling for Precise Nanoparticle Placement: A Route to Wafer-Scale Integration. Nano Letters, 2007. 7(2): p. 439-445.
5. Wright, W.M.D. and D.G. Chetwynd, Can charge writing aid nanotechnological manipulation. Nanotechnology, 1998. 9(2): p. 133-142.

6. Niemeyer, C.M., et al., Site-selective immobilization of gold nanoparticles functionalized with DNA oligomers. Colloid \& Polymer Science, 2001. 279(1): p. 68-72.

7. Rao, S.G., et al., Nanotube electronics: large-scale assembly of carbon nanotubes. Nature (London, United Kingdom), 2003. 425(6953): p. 36-37.

8. Yellen, B.B. and G. Friedman, Programmable assembly of heterogeneous colloidal particle arrays. Advanced Materials, 2004. 16(2): p. 111115.

9. Jacobs, H.O. and G.M. Whitesides, Submicrometer patterning of charge in thin-film electrets. Science, 2001. 291(5509): p. 1763-1766.

10. Barry, C.R., et al., Printing nanoparticle building blocks from the gas-phase using nanoxerography. Appl. Phys. Lett., 2003. 83: p. 5527.

11. Mesquida, P. and A. Stemmer, Attaching silica nanoparticles from suspension onto surface charge patterns generated by a conductive atomic force microscope tip. Advanced Materials (Weinheim, Germany), 2001. 13(18): p. 13951398.

12. Naujoks, N. and A. Stemmer, Micro- and nanoxerography in liquids - controlling pattern definition. Microelectronic Engineering, 2005. 7879: p. 331-337.

13. Krinke, T.J., et al., Positioning of nanometer-sized particles on flat surfaces by direct deposition from the gas phase. Applied Physics Letters, 2001. 78(23): p. 3708-3710.

14. Barry, C.R., J. Gu, and H.O. Jacobs, Charging Process and Coulomb-Force-Directed Printing of Nanoparticles with Sub-100-nm Lateral Resolution. Nano Letters, 2005. 5(10): p. 20782084.

15. Barry, C.R. and H.O. Jacobs, Fringing Field Directed Assembly of Nanomaterials. Nano Letters, 2006. 6(12): p. 2790-2796.

16. Fudouzi, H., M. Kobayashi, and N. Shinya, Assembling $100 \mathrm{~nm}$ scale particles by an electrostatic potential field. Journal of Nanoparticle Research, 2001. 3(2-3): p. 193-200.

17. Welle, A.M. and H.O. Jacobs, Printing of organic and inorganic nanomaterials using electrospray ionization and Coulomb-force-directed assembly. Applied Physics Letters, 2005. 87(December 26): p. 263119.

18. Xia, Y., et al., Unconventional methods for fabricating and patterning nanostructures. Chemical Reviews (Washington, D. C.), 1999. 99(7): p. 1823-1848. 